\title{
Millimeter Per Minute
}

National Cancer Institute

\section{Source}

National Cancer Institute. Millimeter Per Minute. NCI Thesaurus. Code C105507.

A unit of both speed (scalar) and velocity (vector), defined as the distance of one

millimeter traveled per unit time equal to one minute. 